\title{
A New Method for Generating Pore-Network Models of Porous Media
}

\author{
Amir Raoof - S. Majid Hassanizadeh
}

Received: 18 April 2008 / Accepted: 29 April 2009 / Published online: 29 May 2009

(C) The Author(s) 2009. This article is published with open access at Springerlink.com

\begin{abstract}
In this study, we have developed a new method to generate a multi-directional pore network for representing a porous medium. The method is based on a regular cubic lattice network, which has two elements: pore bodies located at the regular lattice points and pore throats connecting the pore bodies. One of the main features of our network is that pore throats can be oriented in 13 different directions, allowing a maximum coordination number of 26 that is possible in a regular lattice in 3D space. The coordination number of pore bodies ranges from 0 to 26, with a pre-specified average value for the whole network. We have applied this method to reconstruct real sandstone and granular sand samples through utilizing information on their coordination number distributions. Good agreement was found between simulation results and observation data on coordination number distribution and other network properties, such as number of pore bodies and pore throats and average coordination number. Our method can be especially useful in studying the effect of structure and coordination number distribution of pore networks on transport and multiphase flow in porous media systems.
\end{abstract}

Keywords Porous media $\cdot$ Pore-network model $\cdot$ Coordination number distribution

\section{Introduction}

Pore-scale processes govern the fundamental behavior of multi-phase multi-component porous media systems. The complexity of these systems, the difficulty to obtain direct pore-scale observations, and difficulties in upscaling the processes have made it difficult to study these systems and to accurately model them with the traditional averaging approaches. Determination of most multiphase properties (e.g., residual saturation) and constitutive

\footnotetext{
A. Raoof $(\varangle) \cdot$ S. M. Hassanizadeh

Universiteit Utrecht, Budapestlaan 4, 3584 CD Utrecht, The Netherlands

e-mail:raoof@geo.uu.nl
}

S. M. Hassanizadeh

e-mail:hassanizadeh@geo.uu.nl 
relations (e.g., capillary pressure, relative permeability) has been based primarily on empirical approaches which are limited in their detail and applicability. Pore-scale modeling (e.g., pore-network and Lattice-Boltzmann model) has been used to improve our descriptions of multiphase systems and to increase our insight into micro-scale flow and transport processes. Their power and versatility lies in their ability to explain macroscopic behavior by explicitly accounting for the relevant physics at the pore level. However, in order to produce realistic predictions, network models require accurate descriptions of the morphology of real porous medium. Previous works have clearly demonstrated the importance of the geometric properties of the porous media, in particular, the distributions of sizes and shapes of pores and throats and the characterization of pore-throat correlations (Larson et al. 1977, 1991; Øren and Pinczewski 1991, 1994; Blunt et al. 1992, 1994; Heiba et al. 1992; Øren et al. 1992, 1994; Ioannidis and Chatzis 1993; Paterson et al. 1996a, b; Pereira et al. 1996; Knackstedt et al. 1998). Equally important to the geometric properties are network topology parameters such as connectivity or coordination number and coordination number distribution. Coordination number, $z$, is defined as the number of bonds (or pore throats) associated to a site (or pore body) in the network.

Pore geometry and topology have a major influence on solute transport and/or multiphase flow in porous systems. For example, in multiphase flow, the nonwetting phase may be trapped if it is completely surrounded by the wetting fluid and in this case, no further displacement is possible in a capillarity-controlled displacement. These isolated nonwetting blobs are at residual saturation and their size distribution and shape can have significant effects on fate and transport of dissolved pollutants (Mayer and Miller 1992; Reeves and Celia 1996; Dillard and Blunt 2000). The nonwetting blobs assume shapes that are influenced by the pore geometry and topology (e.g., aspect ratio, connectivity, and pore-size variability) and their size can range over several orders of magnitude. Sok et al. (2002) have concluded that a more complete description of network topology is needed to accurately predict residual phase saturations. Therefore, it is extremely important to ensure that a pore-network model captures the main features of the pore geometry of porous medium.

The primary topological feature of a network is the coordination number. As has been noted by many authors (Chatzis and Dullien 1977; Wilkinson and Willemsen 1983), the coordination number will influence the flow behavior significantly and also it has a significant impact on the trapping of residual nonwetting phase in multiphase flow; e.g., through bypassing and piston-like pore filling (Fenwick and Blunt 1998; Lake 1989).

A pore network model must represent not only the mean coordination number but also the distribution of the coordination number of the medium. Despite the overwhelming evidence for the presence of a wide range of coordination numbers in real porous media, in most resent network modeling studies, a regular network with a fixed coordination number of six has been used. This means that a given site is connected to six neighboring sites via bonds, which are only located along the lattice axes in three principal directions. This kind of models neglects the topological randomness of porous media, and they also show direction dependence. For example, applying the pressure gradient in diagonal directions, the resulting flow could not happen in these directions since there are no connections in diagonal directions. We refer to this type of network as a three-directional lattice pore-network.

With respect to coordination number, Ioannidis et al. (1997a, b) measured the average coordination number, $\bar{z}$, for serial sections of a sandstone core and for stochastic porous media and found $\bar{z}=3.5$ and $\bar{z}=4.1$ (Ioannidis and Chatzis 2000), respectively. Bakke and coworkers (Bakke and Øren 1997; Øren et al. 1998a, b; Øren and Bakke 2002a, b) developed a process-based reconstruction procedure which incorporates grain size distribution and other petrographical data obtained from 2D thin sections to build network analogs of 
real sandstones. They report mean coordination numbers of $\bar{z}=3.5-4.5$ (Øren and Bakke 2002a, b).

Direct measurements of 3D pore structure using synchrotron X-ray computed microtomography (micro-CT) (Flannery et al. 1987; Dunsmoir et al. 1991; Spanne et al. 1994) coupled with skeletonization algorithms (Thovert et al. 1993; Lindquist et al. 1996; Bakke and Øren 1997; Øren et al. 1998a, b; Øren and Bakke 2002a) indicate that $\bar{z}=4$ for most sandstones.

Arns et al. (2004) did comprehensive study of the effect of network topology on drainage relative permeability. They considered the topological properties of disordered lattice (rock network) derived from a suite of topological images of Fontainbleau sandstone $(\bar{z}=3.3-3.8)$ which displayed a broad distribution of coordination number (Lindquist et al. 2000). Then, they constructed some different network types and compared them to the rock network. The first network type was regular cubic lattice network with fixed coordination number of 6 and identical geometric characteristics (pore and throat size distribution). Comparison between the relative permeability curve for the rock network and that computed on a regular cubic lattice showed poor agreement. Second network type was regular lattice network with average coordination number similar to rock network. This network also showed poor agreement in comparison with rock network. Their result showed that matching the average coordination number of a network is not sufficient to match relative permeabilities. Topological characteristics other than mean coordination number are important in determining relative permeability. The third network was random structure network with coordination number distribution which closely matches that of equivalent rock network. They observed much reasonable approximation to the relative permeability curve. Their results clearly show the importance of matching the full coordination number distribution when generating equivalent network models for real porous media. They have increased the size of network and they found network sizes up to the core scale still exhibit a significant dependence on network topology.

Direct mapping will yield disordered lattice, whereas statistical mapping, for the sake of convenience, is done on regular lattices. There are some studies showing the equivalence of regular and disordered systems (e.g., Arns et al. 2004; Jerauld et al. 1984) with same coordination number distribution. This formally justifies application of work on regular lattices to real porous media.

Studies done by Lindquist et al. (2000) involving the use of high-resolution X-ray computer tomography for imaging the porous media have shown that there is a wide-ranging coordination number in the real porous media. They used images from four different Fontainbleau sandstone samples, with porosities of $7.5 \%, 13 \%, 15 \%$, and $22 \%$ to find geometric and topological quantities required as input parameters for equivalent network models. They found coordination numbers of larger than 20 depending on medium porosity. For example, they found coordination numbers up to 23 at $15 \%$ porosity and 14 at $13 \%$ porosity. The coordination number decreased considerably with decreasing porosity.

Moreover, most of these studies (Ioannidis et al. 1997a, b; Lindquist et al. 2000; Øren and Bakke 2002b) show that the rock samples exhibited a broad distribution of coordination numbers. Whilst the majority of pores were 3-connected, some pores displayed $z>15$ (Ioannidis and Chatzis 2000; Lindquist et al. 2000; Øren and Bakke 2002b). Øren and Bakke (2003) have used information obtained from $2 \mathrm{D}$ thin sections to reconstruct $3 \mathrm{D}$ porous medium of Berea sandstone. They found the coordination number ranging from 1 to 16 with an average value of 4.45 .

In particular, the distribution of $z$ on disordered networks was shown to have a strong effect on the resultant residual phase saturation. Al-Raoush and Willson (2005) have produced 
high-resolution, three-dimensional images of the interior of a multiphase porous system using synchrotron X-ray tomography. The porous medium was imaged at a resolution of $12.46 \mu \mathrm{m}$ following entrapment of the nonwetting phase at residual saturation. Then they extracted the physically representative network structure of the porous medium. They found that the mean coordination number of pore bodies that contained entrapped nonwetting phase was 10.2. This was much higher than the mean coordination number of the system, which was 3.78.

In this study, we present a new method to generate a multi-directional pore network for representing a porous medium. The method is based on a regular cubic lattice network, which has two elements: pore bodies located at the regular lattice points and pore throats connecting the pore bodies. One of the main features of our network is that pore throats can be oriented in 13 different directions, allowing a maximum coordination number of 26 that is possible in a regular lattice in 3D space. The coordination number of pore bodies ranges from 0 to 26 , with a pre-specified average value for the whole network.

\section{Methodology and Formulation}

\subsection{General Network Elements}

Consider a lattice composed of an array of cubes. Let us call the line intersections "sites", and the segments connecting them "bonds". A bond is assumed to exist between each pair of nearest neighbor sites in the lattice. In this multi-directional 3D network, the bonds are aligned in 13 different directions. Figure 1 shows all possible connections for site no. 14 in the center of a $3 \times 3 \times 3$ network, consisting of 8 cubes. Therefore, in a cubic lattice, one bond can be connected to a maximum 50 nearest neighboring bonds. A site can be connected to a maximum of 26 nearest neighbor sites, which is equal to the maximum coordination number, $z=26$.

For the simplicity of description of the network, let us assume that the flow is from left to the right, determined by the macroscopic pressure gradient. Denote the number of lattice

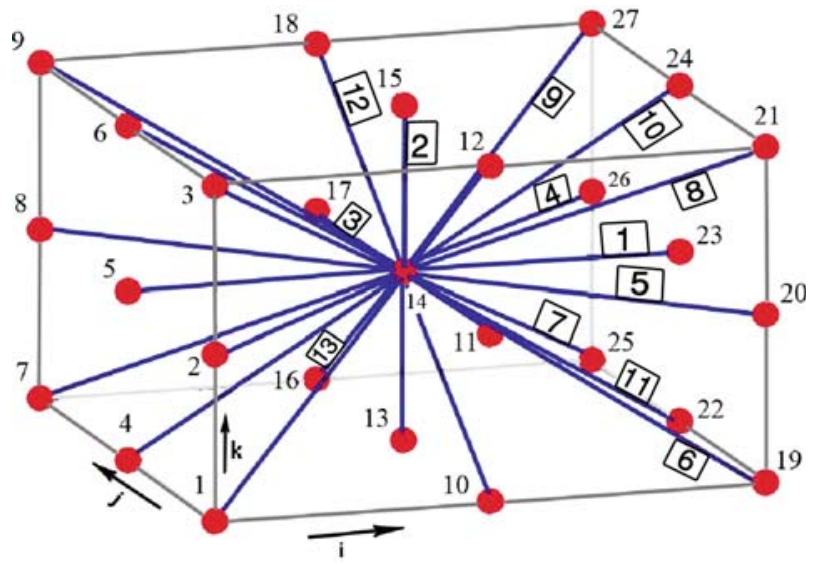

Fig. 1 Network consisting of 8 cubes, size: $N_{i}=3, N_{j}=3, N_{k}=3$. Numbers inside the squares show bonds' directions and others are site numbers. To keep the figure less crowded, only bonds which are connecting site 14 to its neighboring sites are shown 
sites in the flow direction by $N_{i}$, and let $N_{j}$ and $N_{k}$ denote the number of sites in the other two directions. Thus, the size of a 3D network is $N_{i j k}\left(=N_{i} \times N_{j} \times N_{k}\right)$.

Let $N_{j k}\left(=N_{j} \times N_{k}\right)$ be the size of a two-dimensional rectangular grid perpendicular to overall flow direction. Choice of the numbering of lattice bonds directions is not critical; in Fig. 1, directions 1, 2, and 3 are chosen to be the three principal directions.

Note that, to keep the figure less crowded, we have shown lattice bonds passing through site 14 only. All other lattice bonds in network receive the same direction number as their parallel bond in the Fig. 1.

The system of numbering of lattice is shown in Fig. 1: starting from the first vertical plane at left, within each $N_{j k}$ plane, sites are numbered from bottom right to top left and then continued with the next $N_{j k}$ plane in the overall flow direction.

A given site can be connected to two other sites along any given direction number: one in forward direction (increasing site number) and one in the backward direction (decreasing site number). For example, in the direction no. 1, site 14 is connected forwardly to site no. 23 and backwardly to site no. 5 .

Obviously, sites which are located on the boundaries have smaller coordination numbers. For example, in Fig. 1, the only site with all connections in all the 13 directions is site number 14 , since the rest of sites are situated on the boundaries of the network.

\subsection{Network Connections}

Based on the numbering system described above and shown in Fig. 1, we can provide expressions for determining the connections between various sites inside a general network. Table 1 gives expressions for finding sites connected to site number " $N$ " in forward directions. As an illustration, the site numbers connected to site $N=14$ in Fig. 1 are also given.

Assuming horizontal overall flow direction, we also assume that sites in the left and right boundaries cannot connect to other sites in the same plane, but can only connect to sites

Table 1 Expressions for finding all sites connected to site number $N$ in the forward directions. In example of Fig. $1, N_{k}=3$ and $N_{j k}=9$

\begin{tabular}{lll}
\hline $\begin{array}{l}\text { Direction } \\
\text { number }\end{array}$ & $\begin{array}{l}\text { General expression } \\
\text { for site: } N\end{array}$ & $\begin{array}{l}\text { Example of } \\
\text { Fig. } 1, N=14\end{array}$ \\
\hline 1 & $N+N_{j k}$ & 23 \\
2 & $N+1$ & 15 \\
3 & $N+N_{k}$ & 17 \\
4 & $N+N_{j k}+N_{k}$ & 26 \\
5 & $N+N_{j k}-N_{k}$ & 20 \\
6 & $N+N_{j k}-N_{k}-1$ & 19 \\
7 & $N+N_{j k}+N_{k}-1$ & 25 \\
8 & $N+N_{j k}-N_{k}+1$ & 21 \\
9 & $N+N_{j k}+N_{k}+1$ & 27 \\
10 & $N+N_{j k}+1$ & 24 \\
11 & $N+N_{j k}-1$ & 22 \\
12 & $N+N_{k}+1$ & 18 \\
13 & $N+N_{k}-1$ & 16 \\
\hline
\end{tabular}


(a)

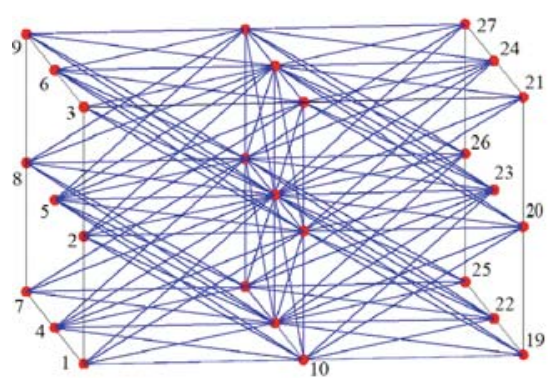

(b)

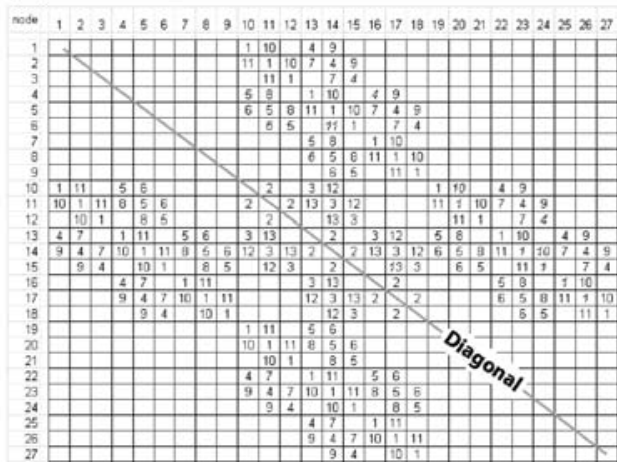

Fig. 2 Network with full connections with the size: $N_{i}=3, N_{j}=3, N_{z}=3$. a Configuration of the network (site numbers are shown for some nodes and system of numbering is the same as in Fig. 1). b The corresponding connection matrix. Empty boxes indicate that no connection between corresponding sites exists

inside the network. Due to applying this restriction, assigning different pressure values to different sites in the left or right boundaries would not cause any in-plane flow.

\subsection{Connection Matrix}

Perhaps, the best way to get a desired pattern in the network, to generate the bonds, and understanding the effect of network boundaries on these bonds is to examine the matrix of connections. Figure 2 shows a fully connected network of size $N_{i}=N_{j}=N_{k}=3$ and the corresponding connection matrix.

The connection matrix is a centrosymmetric matrix (symmetric with respect to both the main diagonal and the anti-diagonal) with the size: $N_{i j k} \times N_{i j k}(27 \times 27$ in the example of Fig. 2). As shown in Fig.2(b), row or column number is a site number. Matrix entries are direction numbers. An entry has a nonzero value only if the corresponding sites (given by row and column numbers) are connected to each other in the network.

Blank entries in the connection matrix correspond to sites which are not connected to each other or are the effect of network boundaries. The total number of nonzero entries in each row is the coordination number for the site number corresponding to that row. Clearly, coordination number is maximum $(z=26)$ for sites inside a network and has a smaller value for sites on the boundaries. Since in this example, site 14 is the only site with all 26 connections, only row 14 has 26 nonzero entries.

In the connection matrix, the upper triangle (above the diagonal line in Fig. 2(b)) contains forward connections of the network and the lower triangle contains backward connections. For example, site number 10 is connected to sites 1, 2, 4, and 5 in backward direction and to sites $11,13,14,19,20,22$, and 23 in the forward direction. Note that all entries of a minor diagonal have the same value, which is a lattice direction number. Because the connection matrix is always symmetric, we only need to keep the upper triangle which has 13 minor diagonals. Furthermore, the connection matrix, is a sparse matrix, and we can convert the full matrix to a compact form by squeezing out all zero elements. 


\subsection{Elimination Process}

Since real porous media are topologically random with a wide distribution of coordination numbers, we generate random networks with topological properties which can be specified arbitrarily. These networks can provide a better match to real porous media topology than regular networks with a fixed coordination number. This results in a random network, which can match both the mean and the distribution of coordination numbers of a porous medium.

The coordination number of 26 is much larger than the commonly observed mean value of coordination numbers. To have a smaller mean coordination number and a desired coordination number distribution, we should eliminate some bonds in the network. It is worth mentioning that, after elimination process, the connection matrix is no longer centrosymmetric and it is only a symmetric sparse matrix. There are many ways to eliminate some of bonds (see e.g., Arns et al. 2004). In general, one may follow a random or a regular elimination procedure. We have chosen the former approach and have formulated a consistent and flexible procedure described below.

Suppose that each bond may exist in only two possible states: 'open' or 'blocked'. We further assume that each bond's state is random and independent of its neighbors. We then assign a set of 13 threshold numbers, $p_{i}, i=1,2, \ldots, 13$, one for each direction. Each $p_{i}$ value is between zero and one and denotes the probability of having a bond in direction $i$. Next, during network construction (e.g., putting the connection matrix together), for each and every possible bond, we generate a random number with uniform distribution, also between zero and one, which we call elimination number. If this elimination number is greater than the threshold number of the corresponding direction, the bond's state is assigned to be blocked; otherwise it will be set to open. So, the larger the threshold number, $p_{i}$, the more the chance to have an open bond in direction number $i$. There are two limiting cases: (a) If the threshold number $p_{i}$ is equal to 1.0, then all the bonds along lattice direction $i$ will be open; (b) If the threshold number $p_{i}$ is equal to zero it means all bonds along that lattice direction $i$ will be blocked, indicating no connectivity along that direction. By putting $p_{1}=p_{2}=p_{3}=1.0$ (direction numbers: 1, 2, 3 in Fig. 1) and zero for the other 10 directions, we will end up with the commonly used regular network with $z=6.0$ with connections only in three principal directions.

If we choose the same value, $\Pi$, for all the threshold numbers, say $p_{1}=p_{2}=\cdots=\Pi$, then as $\Pi$ increases from zero, more and more sites become connected into one giant cluster. At some point, the connected cluster spans the entire lattice both vertically and horizontally. The threshold number at which this happens (approximately 0.08 for our cubic lattice network with the size: $30 \times 30 \times 30$ ) is called the critical probability, $p_{\mathrm{c}}$, also known as the cubic lattice bond percolation threshold. This elimination procedure varies directly with the proportion of open bonds (not the sites), so it is called bond percolation (Berkowitz and Ewing 1998). If we realize that the fluid can flow only through bonds which are "open", then below the $p_{\mathrm{c}}$ value the lattice will have zero conductivity, while above the $p_{\mathrm{c}}$, the conductivity will rise as the $p_{i}$ increases. Hence, there is a strong relation between connectivity of the elements (the so-called microscopic properties) and the physical properties of the entire system (or the so-called macroscopic properties).

There are some observations which have also been made in earlier studies (e.g., Berkowitz and Ewing 1998). First, as the proportion of open sites increases, the proportion of blocked sites that have open neighbors also increases. Second, once $\Pi>p_{\mathrm{c}}$, we will find that some bonds can have fluid flowing through them (the ensemble of these bonds is called the "backbone" of the network), while others are simply isolated clusters or deadend bonds; the proportion of these branches varies as a function of $\Pi$. Third, the clusters 
grow larger (and merge) with an increase in П. The reverse happens to the blocked bonds; they reduce in number and become more and more isolated as the probability of open sites increases.

We can generate a network with a pre-specified mean coordination number, $\bar{z}$, by choosing a common threshold number $\Pi=\bar{z} / 26$ for all directions. For example, we can generate a network with the mean coordination number of 6 by choosing $\Pi=6 / 24=0.231$ for all directions. Obviously, the coordination number distribution still ranges from 0 to 26 for any given $\bar{z}$.

When threshold numbers are chosen to be all different, we can create different connectivities in different directions. This can result in anisotropic lattices. For example, Friedman and Seaton (1996) considered anisotropic lattices. They found that permeability and diffusive properties depend strongly on anisotropy induced by directionally different coordination numbers, and by anisotropic pore size distributions.

It is also possible to assign correlated elimination numbers in various directions. The correlation length, the range over which status of one bond is correlated with (or influenced by) status of other bonds, can be chosen based on desired lattice spacings or prescribed size of pore bodies. This allows us to create an anisotropic network with more connections, and as a result a larger permeability in certain directions. In an elaborate simulation study, Jerauld and Salter (1990) found that, as the degree of correlation between nearby bonds increased, saturated permeability increased. An explanation for this increase in saturated permeability with increased correlation can be found in Ambegaokar et al. (1971) model of hopping conductivity, which holds that flow through a random medium is dominated by a few pathways of high conductivity. Correlation appears to increase the probability and/or the conductance of some of individual pathways (Jerauld and Salter 1990). Also, Renault (1991) found that as the correlation length was increased from 0 to 5 times that of the site spacing, the percolation threshold decreased. In our study, in the test cases of Sect. 3, we have used different threshold numbers for different directions. However, the generated elimination numbers have been uncorrelated.

Another approach to reduce coordination number from 26 to a pre-specified value is to choose a regular elimination pattern. For example, we may assign blocked states for two successive bonds and open state for third bond and then repeat this pattern for all the directions. We can also apply combination of random and regular patterns to obtain specific connectivities and coordination number distributions for the network.

\subsection{Isolated Clusters and Dead-End Bonds}

We now proceed to the issue of conduction through the bonds. Obviously, flow problems are of interest only for $\Pi>p_{c}$. Note that, after elimination process, many of the connected bonds will not conduct flow, since neither they belong to the percolating cluster nor they form dead-end pores. Examples of possible situations are shown in Fig. 3, where dead-end bonds are shown as hollow circles crossed by a line, and isolated sites and isolated clusters are marked by hollow circles and squares, respectively. The network backbone does not include isolated sites and clusters. Isolated sites and clusters also may cause numerical problems since they lead to a singular or ill-conditioned coefficient matrix for simulating flow within the network. Therefore, we should find all the isolated sites and clusters and eliminate them from our network. Regarding dead-end pores, we may choose to omit them if they are not important for a given process (such as flow), or keep them for processes where they pay a role (such as solute transport with diffusion). 
Fig. 3 Example of eliminated sites, isolated clusters, and dead-end bonds

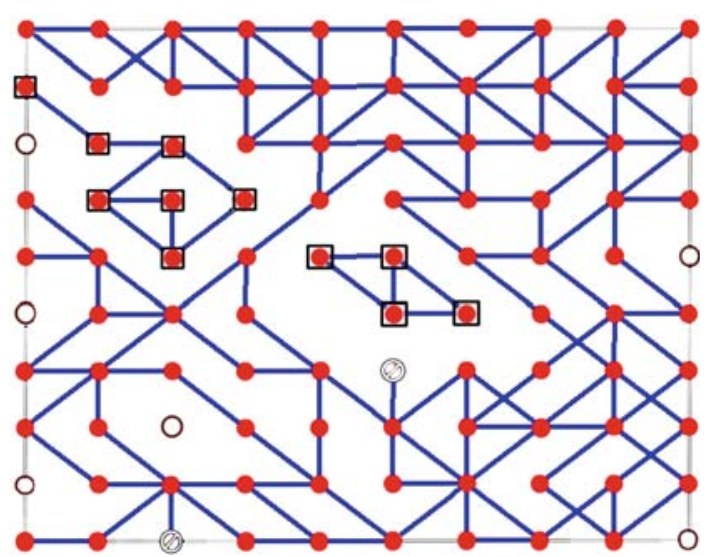

It is worth mentioning that one can avoid this situation (isolated clusters and dead-end pores) through the choice of threshold number. For example, we can put $p_{1}=1.0$ so that all the bonds in the overall flow direction are open. And then we can apply elimination to the other directions.

Dead-end sites (sites with only one bond connected to them) have only one nonzero value in their corresponding row index in the connection matrix. So, we can find them fairly easily and eliminate them from the connection matrix or we can keep them as the dead-end pores of porous media.

Finding and eliminating isolated clusters is not as easy as in the case of dead-end sites. To do so, we employ a search algorithm to find "backbone". We start from one of the boundaries across which flow is allowed. For each and every site on that boundary, we apply a search algorithm to find all the sites connected to it directly or through other sites. At the end, if we reach to another flow boundary, it means that this group of sites forms a backbone. After finding the backbone, we eliminate all other bonds as they belong to isolated clusters. To do tracking, it is crucial to have an efficient search algorithm. We have employed an algorithm based on the frequency of bonds. The details of our algorithm are given in Appendix A.

Looking at the topology of the resulting network, our network is of semi-regular type since after the elimination process, we do not always have a site at each lattice point. In fact, lattice points do not even need to be exactly in regular cubic pattern, and we have freedom to shift them away from the lattice point and/or move them into the position of eliminated pores or clusters, given that they would not have any contact with any other neighboring site (Fig. 4). This feature along with different pore body sizes (thus, different bond lengths) makes this network a semi-irregular type network. However, since the random network is a subset of a regular network (e.g., the cubic lattice network), sites are only connected to their nearest neighbors and the network contains no long bonds. Arns et al. (2004) considered the effect of disordered topology on relative permeability. They generated diluted networks with a disordered topology and $\bar{z}=4$, where the positions of the pore centers of the sample were preserved during the network construction. Then, they compared relative permeabilities for these networks with those for a regular network. The effect of topological disorder on relative permeability was minor. Their result is in agreement with the findings of Jerauld et al. (1984) who showed that disordered topology had little effect on the percolation and conduction properties of networks. The equivalence of regular and disordered systems formally justifies application of work on regular lattices to real porous media. 
Fig. 4 A network of size: $N_{i}=10, N_{j}=1, N_{k}=10$. Isolated clusters are eliminated and locations of some sites are modified to get an irregular lattice

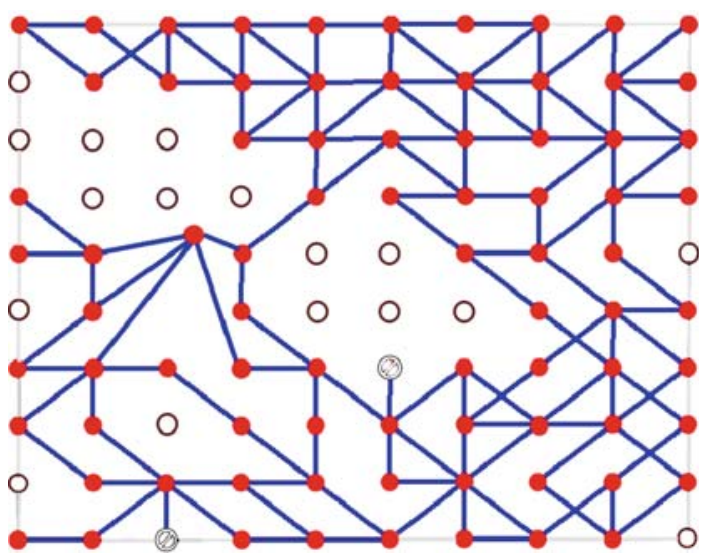

Table 2 Properties of the generated network and those reported by Al-Kharusi and Blunt (2006)

\begin{tabular}{lll}
\hline Characteristic & $\begin{array}{l}\text { Data reported by } \\
\text { Al-Kharusi and Blunt (2006) }\end{array}$ & $\begin{array}{l}\text { Generated } \\
\text { model }\end{array}$ \\
\hline Number of pores & 4997 & 5030 \\
Number of bonds & 8192 & 7605 \\
Mean coordination number & 3.2 & 3.03 \\
Number of bonds to inlet & 227 & 238 \\
Number of bonds to outlet & 206 & 219 \\
porosity $(\%)$ & 13.6 & 13.5 \\
\hline
\end{tabular}

\section{Test Cases (Optimization Using Genetic Algorithm)}

In order to illustrate the applicability and versatility of our method, we have employed it to generate two different networks representing a consolidated porous medium, and a granular soil sample. In principle, through optimization process, one can match various characteristics such as coordination number distribution, average coordination number, number of pores, number of bounds, etc. In the test cases presented here, we have chosen to match the coordination number distribution of the porous media under this study. Thus, threshold numbers, $p_{i}$, are the variables to be optimized. The generated elimination numbers have been chosen to be uncorrelated.

Other porous media characteristics (listed in Table 2) have been used to verify the resulting networks after optimization. The optimization is based on minimizing the objective or fitness function. The sum of the absolute values of differences between coordination number distribution of the real porous medium and generated network was used as the fitness function. We have employed genetic algorithm (GA) for the purpose of optimization. Genetic algorithms have been used to solve difficult problems with objective functions that are not well behaved, i.e., they do not possess properties such as continuity, differentiability, etc. (Davis 1991; Goldberg 1989; Holland 1975; Michalewicz 1994; Houck et al. 1995).

The genetic algorithm provides a method for solving both constrained and unconstrained optimization problems. In our case, the valid range for threshold numbers is between zero and one. However, to prevent generation of networks with huge variations in connectivities 


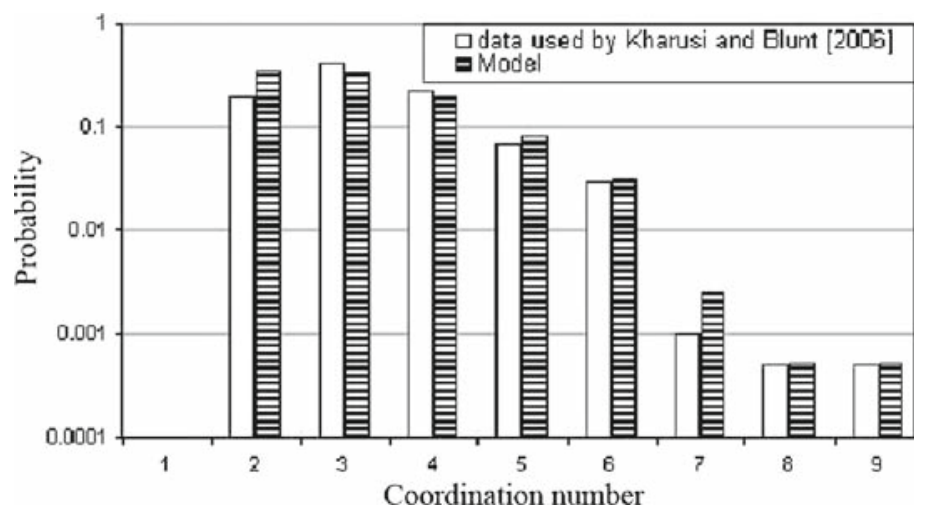

Fig. 5 Comparison between coordination number distributions from real porous media and generated network

in different directions, we have changed the upper bound constraint to 0.30 . By enforcing this condition, we make sure that the generated networks have acceptable connectivities (without meaningless topologies). Genetic algorithm differs from more traditional search algorithms in that it works with a number of candidate solutions (population) rather than just one solution. The algorithm begins by creating a random initial population. Then at each step, it selects individuals at random from the current population and uses them to produce the next generation. Over successive generations, the population "evolves" toward an optimal solution (for details of the method, readers are referred to Houck et al. 1995). We have chosen the initial population size of 70 . In order to minimize the objective function, genetic algorithm produced 200 successive generations. The results of optimization are shown in the following sections.

\subsection{Generating a Consolidated Porous Medium (Sandstone Sample)}

For this test case, we have chosen data reported by Al-Kharusi and Blunt (2006) on a Fontainebleau sandstone sample. They have utilized the concept of "maximal balls" (Silin et al. 2003) to compute the locations and sizes of pores and throats and to create a topologically equivalent representation of $3 \mathrm{D}$ images.

As mentioned above, a representative network was created through optimizing threshold numbers, $p_{i}$, such that coordination number distribution of the resulting network matches the measured coordination number distribution of the sample. These are both shown and compared in Fig. 5. The optimization was performed through 200 generations using genetic algorithm.

After optimization, to verify the accuracy of the resulting network, various geometrical characteristics of the generated network were compared with those of the sandstone sample, reported in Table 2. It is evident that there is a good agreement between various characteristics of two networks.

After generating the network, we used it as the skeleton and assigned site and bond size distributions to match the measured porosity. The radii of sites were given by an uncorrelated truncated lognormal probability distribution and the radii of bonds were determined by size of the sites which are located at the two ends of each bond. This scheme was adopted from Acharya et al. (2004) and used by Joekar-Niasar et al. (2007). The resulting porosity is shown in Table 2. 


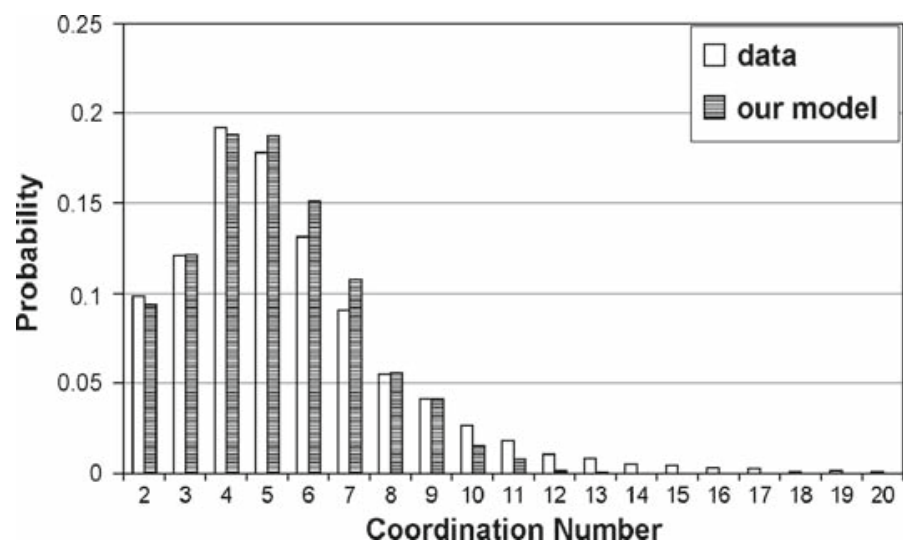

Fig. 6 Comparison between coordination number distributions from granular porous media and generated network

Table 3 Properties of the generated network and those extracted by Thompson et al. (2006)

\begin{tabular}{lll}
\hline Characteristics & $\begin{array}{l}\text { Physical } \\
\text { representative } \\
\text { network }\end{array}$ & $\begin{array}{l}\text { Generated } \\
\text { model }\end{array}$ \\
\hline Number of pores & 4564 & 4056 \\
Number of bonds & 12534 & 10674 \\
Average coordination number & 5.4 & 4.7 \\
Number of bonds to inlet & 214 & 232 \\
Number of bonds to outlet & 196 & 238
\end{tabular}

\subsection{Generating a Granular Porous Medium}

For this test case, we have chosen data on coordination distribution of a granular soil. The method to extract the topological information can be found in Thompson et al. (2006). They presented a new algorithm for extracting topological information of powders and granular materials from high-resolution binary volume data to get a vast amount of morphologic information such as size distribution, porosity, particle aspect ratio, coordination number, and more.

According to the procedure explained in Sect. 3.1, we have generated a pore network representing of the sample through optimizing threshold numbers, $p_{i}$. The coordination number distribution of the granular sample and the resulting network are shown and compared in Fig. 6. An independent measure of accuracy of our resulting network is provided by comparing various characteristics of the generated network with soil sample (Table 3). There is a good agreement between different characteristics of two networks.

\section{Conclusions}

We have presented a new method to generate 3D network representing porous media with coordination number up to 26 . We used distribution of coordination number and applied 
the same into multi-directional cubic lattice network rather than using direct mapping into a physically representative disordered network (e.g., based on the X-ray tomographic images).

This new method is straightforward to facilitate programming and fast resulting in reduced running time. For example, for a network with size $N_{i}=300, N_{j}=100$, and $N_{k}=100$, and the average coordination number 6 , CPU time of $20 \mathrm{~s}$ with Intel $2.40 \mathrm{GHz}$ and $2.00 \mathrm{~GB}$ of RAM is needed to generate the network and detect the dead-end bonds and isolated clusters.

It is possible to construct networks with regular or random connection structures with a wide range of coordination number distribution as well as different directional connectivities. A quantitative comparison between properties of our generated network and those of consolidated and granular porous media samples shows that intrinsic properties, such as mean connectivity, coordination distribution, and porosity, are adequately captured in the reconstruction by this semi-regular multi-directional network. An effective optimization process using genetic algorithm was employed to reproduce the coordination number distributions. The coordination number distributions of the generated networks and the real porous media are in good agreement showing genetic algorithm may be a promising and effective approach for optimization purpose in pore network.

\section{Appendix A: Search Algorithm}

In order to find the isolated clusters, we need to employ tracking. We have employed an algorithm based on the frequency of bonds. In order to illustrate the procedure, considering the network of Fig. 2, we randomly eliminated some bonds by changing the states of them from open to block in the connection matrix of Fig. 2. Figure A.1 shows the network configuration after this random elimination.

The connection matrix is a sparse matrix. Hence, we convert it in to compact form. This results in a matrix of the size $3 \times N_{\text {bond }}$ ( $N_{\text {bond }}=$ number of bonds). Table A.1 shows the corresponding compact matrix for upper triangle part of connection matrix belonging to Fig. A.1. In the compact form shown in Table A.1, the first row (ROW in Table A.1) contains the row index of original sparse matrix, the second row (COL) contains the column index, and the third row (Entry) contains the corresponding nonzero direction numbers. Since this

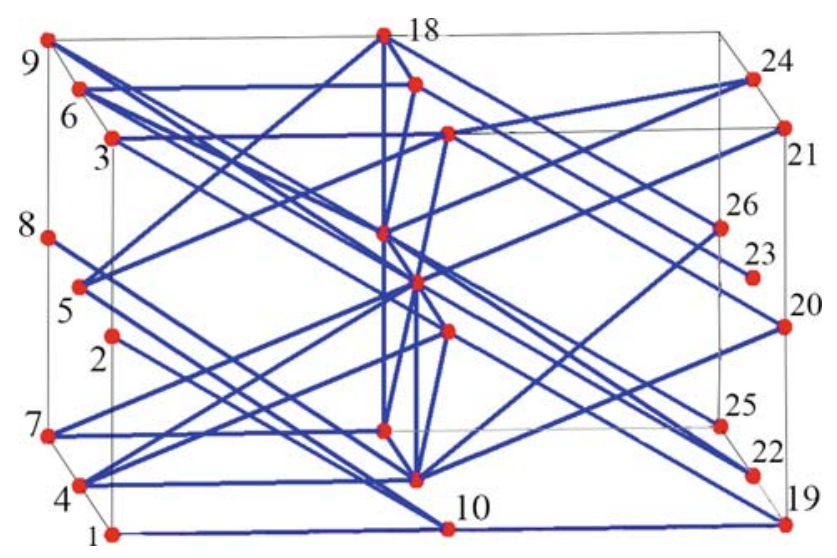

Fig. A.1 A network of size: $N_{i}=3, N_{j}=3, N_{k}=3$ after random elimination. Site numbers are shown for some nodes; system of numbering is the same as in Fig. 1 . $N_{\text {bond }}=42, N_{\text {site }}=26$ 
Table A.1 Compact form of connection matrix for example of Fig. A.1

\begin{tabular}{|c|c|c|c|c|c|c|c|c|c|c|c|c|c|c|c|c|c|c|c|c|}
\hline ROW 1 & 2 & 3 & 3 & 4 & 4 & 4 & 5 & 5 & 5 & 6 & 6 & 6 & 7 & 7 & 8 & 9 & 9 & 10 & 11 & 11 \\
\hline COL 10 & 10 & 12 & 11 & 13 & 11 & 14 & 10 & 12 & 18 & 15 & 17 & 14 & 16 & 14 & 13 & 14 & 17 & 19 & 14 & 19 \\
\hline Entry 1 & 11 & 1 & 11 & 1 & 8 & 10 & 6 & 8 & 9 & 1 & 7 & 11 & 1 & 8 & 6 & 6 & 11 & 1 & 3 & 11 \\
\hline ROW 11 & 12 & 12 & 12 & 13 & 13 & 13 & 13 & 14 & 14 & 14 & 14 & 15 & 15 & 15 & 16 & 17 & 17 & 17 & 17 & 18 \\
\hline COL 13 & 24 & 20 & 14 & 14 & 16 & 20 & 26 & 17 & 21 & 22 & 16 & 18 & 23 & 17 & 17 & 18 & 22 & 24 & 25 & 26 \\
\hline Entry 13 & 4 & 11 & 13 & 2 & 3 & 8 & 9 & 3 & 8 & 11 & 13 & 3 & 11 & 13 & 2 & 2 & 6 & 8 & 11 & 11 \\
\hline
\end{tabular}

Table B.1 Frequency and cumulative matrix of the forward connections

\begin{tabular}{|c|c|c|c|c|c|c|c|c|c|c|c|c|c|c|}
\hline Site no. & 1 & 2 & 3 & 4 & 5 & 6 & 7 & 8 & 9 & 10 & 11 & 12 & 13 & 14 \\
\hline Frequency & 1 & 1 & 2 & 3 & 3 & 3 & 2 & 1 & 2 & 1 & 3 & 3 & 4 & 4 \\
\hline Cumulative & 1 & 2 & 4 & 7 & 10 & 13 & 15 & 16 & 18 & 19 & 22 & 25 & 29 & 33 \\
\hline Site no. & 15 & 16 & 17 & 18 & 19 & 20 & 21 & 22 & 23 & 24 & 25 & 26 & 27 & \\
\hline Frequency & 3 & 1 & 4 & 1 & 0 & 0 & 0 & 0 & 0 & 0 & 0 & 0 & 0 & \\
\hline Cumulative & 36 & 37 & 41 & 42 & 42 & 42 & 42 & 42 & 42 & 42 & 42 & 42 & 42 & \\
\hline
\end{tabular}

is compact form of connection matrix, it means that site numbers in ROW are connected in the forward direction to site numbers in COL. For example, first column of Table A.1 indicates that site number 1 is connected in the forward direction to site number 10 along lattice direction number 1 .

As we can see, the site numbers in the first row (ROW) of Table A.1 are sorted in increasing order. The number of times a site number appears in ROW of Table A.1 corresponds to the number of forward connections of that site. This information in taken up in a frequency table (see second row of Table B.1) together with its cumulative number of bonds (see third row of Table B.1).

Table B.1 along with second row of Table A.1 can be used to determine to which sites a given site, $N$, is connected based on the following formula:

Forward sites for site $N=\operatorname{COL}(\operatorname{cumulative}(N)-\operatorname{frequency}(N)+1$ :cumulative $(N))$

For example, for site number $12(N=12)$ :

$$
\begin{aligned}
\text { Forward sites for site } 12 & =\operatorname{COL}(\text { cumulative }(12)-\text { frequency }(12)+1 \text { :cumulative }(12)) \\
& =\operatorname{COL}(25-3+1: 25)=\operatorname{COL}(23: 25)=[24,20,14]
\end{aligned}
$$

Hence, the sites to which site number 12 is connected forward are located in position 23-25 in the COL of Table A.1 which are sites number 24, 20, and 14.

To find the backward connections, we need to replace ROW and COL of Table A.1 with each other (since in the connection matrix backward connections are transpose of forward connections) and then sort the matrix in this new ROW and next calculate the frequency vector in the same manner as before. For example, for site 12, we will find that backward sites for site 12 are sites 3 and 5. Hence, site number 12 is connected overall to sites number $24,20,14,3$, and 5. In the next step, we can apply this formula again for each of these sites to find their connections and repeat this tracking. These sites together are one group. At the end, if there is at least one site from right boundary in this group, it means the sites belonging to this group are not isolated clusters; on the other hand, they are isolated clusters and we should delete the whole group from connection matrix.

It is worth mentioning that after construction of frequency vectors (for forward and backward directions) no longer we need ROW in Table A.1. Also we do not need to record entry 
because if we know the connection of two sites then we can calculate the direction number according to formulas in Table 1 to see which of the formulas in Table 1 can produce this connection.

Hence, by recording only COL and frequency vector, we have all informations about connections in the network and we can track connections. In addition, the size of COL is $N_{\text {bond }}$ while the size of frequency vector is $N_{\text {site }}$ ( $=$ number of sites) which is much less than $N_{\text {bond }}$. Hence, using the concept of frequency vector in tracking, first of all, we will have a formula to exactly find the connections for a given site which makes the tracking very fast (in comparison to searching the connection matrix to find specific sites). Second, we will decrease the size of matrices which, in turn, will allow us to use less memory.

Using this algorithm, like the tracking example we have shown above, we can track the sites and get rid of isolated clusters to have the backbone with sites which are connected to both of boundaries.

Acknowledgments Valuable discussions with Prof. Toon Leijnse are acknowledged. We also thank Prof. Ruud Schotting (Utrecht University) for his useful comments. As the members of the International Research Training Group NUPUS, financed by the German Research Foundation (DFG) and the Netherlands Organization for Scientific Research (NWO), the authors thank the DFG (GRK 1398) and NWO (DN 81-754) for their valuable support.

Open Access This article is distributed under the terms of the Creative Commons Attribution Noncommercial License which permits any noncommercial use, distribution, and reproduction in any medium, provided the original author(s) and source are credited.

\section{References}

Acharya, R.C., van der Zee, S.E.A.T.M., Leijnse, A.: Porosity-permeability properties generated with a new 2-parameter 3D hydraulic pore-network model for consolidated and unconsolidated porous media. Adv. Water Resour. 27, 707-723 (2004). doi:10.1016/j.advwatres.2004.05.002

Al-Kharusi, A.S., Blunt, M.J.: Permability prediction and network extraction from pore space images. Paper presented at CMWR XVI-Compitational Methods in Water Resources. Copenhagen, Denmark, June (2006). http://proceedings.cmwr-xvi.org/contributionDisplay.py?contribId=293\&amp; sessionId=9\&amp; confId=a051

Al-Raoush, R.I., Willson, C.S.: A pore-scale investigation of a multiphase porous media system. J. Contam. Hydrol. 77, 67-89 (2005). doi:10.1016/j.jconhyd.2004.12.001

Ambegaokar, V., Halperin, B.I., Langer, J.S.: Hopping conductivity in disordered systems. Phys. Rev. B 4, 2612-2620 (1971). doi:10.1103/PhysRevB.4.2612

Arns, J.-Y., Robins, V., Sheppard, A.P., Sok, R.M., Pinczewaski, W.V., Knackstedt, M.A.: Effect of network topology on relative permeability. Transp. Porous Media 55, 21-46 (2004). doi:10.1023/B:TIPM. 0000007252.68488 .43

Bakke, S., Øren, P.E.: 3-D pore-scale modelling of sandstones and flow simulations in the pore networks. SPE J. 2, 136-149 (1997). doi:10.2118/35479-PA

Berkowitz, B., Ewing, R.P.: Percolation theory and network modeling applications in soil physics. Surv. Geophys. 19, 23-72 (1998). doi:10.1023/A:1006590500229

Blunt, M., King, M.J., Scher, H.: Simulation and theory of twophase flow in porous media. Phys. Rev. A 46, 7680-7699 (1992). doi:10.1103/PhysRevA.46.7680

Blunt, M., King, M.J., Zhout, D.: What determines residual oil saturation in three phase flow. In: Proceedings of the SPE/DOE 9th Symposium on Improved Oil Recovery, Tulsa, OK (1994)

Chatzis, I., Dullien, F.A.L.: Modelling pore structures by 2D and 3D networks with application to sandstones. Can. J. Petroleum Technol, 97-108 (1977) (January-March)

Davis, L.: The Handbook of Genetic Algorithms. Van Nostrand Reingold, New York (1991)

Dillard, L.A., Blunt, M.J.: Development of a pore network simulation model to study nonaqueous phase liquid dissolution. Water Resour. Res. 36(2), 439-454 (2000). doi:10.1029/1999WR900301

Dunsmoir, J.H., Ferguson, S.R., D’Amico, K.L., Stokes, J.P.: X-ray microtomography: a new tool for the characterization of porous media. SPE 22860. In: Proceedings of the 1991 SPE Annual Technical Conference and Exhibition, Dallas, October 6-9 1991 
Fenwick, D.H., Blunt, M.J.: Three-dimensional modeling of three phase imbibition and drainage. Adv. Water Resour. 21(2), 121-143 (1998). doi:10.1016/S0309-1708(96)00037-1

Flannery, B.P., Deckman, H.W., Roberge, W.G., D’Amico, K.L.: Three-dimensional X-ray microtomography. Science 237, 1439-1444 (1987). doi:10.1126/science.237.4821.1439

Friedman, S.P., Seaton, N.A.: On the transport properties of anisotropic networks of capillaries. Water Resour. Res. 32, 339-347 (1996). doi:10.1029/95WR02830

Goldberg, D.: Genetic Algorithms in Search, Optimization, and Machine Learning. Addison_Wesley (1989)

Heiba, A., Sahimi, M., Scriven, L., Davis, H.: Percolation theory of two-phase relative permeability. SPE Reserv. Eng. 7, 123-132 (1992)

Holland, J.: Adaptation in natural and artificial systems. The University of Michigan Press, Ann Arbor (1975)

Houck, C.R., Joines, J.A., Kay, M.G.: A Genetic Algorithm for Function Optimization: A Matlab Implementation. NCSU-IE TR 95, 09 (1995)

Ioannidis, M.A., Chatzis, I.: Network modelling of pore structure and transport properties of porous media'. Chem. Eng. Sci. 48, 951-972 (1993). doi:10.1016/0009-2509(93)80333-L

Ioannidis, M.A., Chatzis, I.: On the geometry and topology of 3D stochastic porous media. J. Colloid Interface Sci. 229, 323-334 (2000). doi:10.1006/jcis.2000.7055

Ioannidis, M.A., Kwiecien, M.J., Chatzis I., MacDonald, I.F. and Dullien, F.A.L.: Comprehensive pore structure characterization using 3-D computer reconstruction and stochastic modeling. Technical report, SPE Paper 38713 (1997a)

Ioannidis, M.A., Kwiecien, M.J., Chatzis, I., MacDonald, I.F., Dullien, F.A.L.: Comprehensive pore structure characterization using 3D computer reconstruction and stochastic modeling. In: Proceedings of the SPE Annual Technical Conference and Exhibition, San Antonio, TX (1997b)

Jerauld, G.R., Salter, S.J.: The effect of pore-structure on hysteresis in relative permeability and capillary pressure: pore-level modelling. SPE Transp. Porous Media 5, 103-151 (1990). doi:10.1007/BF00144600

Jerauld, G., Scriven, L., Davis, H.: Percolation and conduction on the 3D Voronoi and regular Networks: a second case study in topological order. J. Phys. Chem. 17, 3429 (1984)

Joekar-Niasar, V., Hassanizadeh S.M., Leijnse A.: Insights into the relationships among capillary pressure, saturation, interfacial area and relative permeability using pore-network modeling. Transp. Porous Med. (2007). doi:10.1007/s11242-007-9191-7

Knackstedt, M.A., Sheppard, A.P., Pinczewski, W.V.: Simulation of mercury porosimetry on correlated grids: evidence of extended correlated heterogeneity at the pore scale in rocks. Phys. Rev. E. Rapid Commun. 58, R6923-R6926 (1998)

Lake, L.W.: Enhanced oil recovery. In: Larson, R.G., Scriven, L.E., Davis, H.T. (eds.) Percolation Theory of Residual Phases in Porous Media. Prentice Hall, Englewood Cliffs, NJ (1989)

Larson, R., Scriven, L.E., Davis, H.T.: Percolation theory of residual phases in porous media. Nature 268, 409-413 (1977). doi:10.1038/268409a0

Larson, R., Scriven, L.E., Davis, H.T.: Percolation theory of two-phase flow in porous media. Chem. Eng. Sci. 36, 57-73 (1991). doi:10.1016/0009-2509(81)80048-6

Lindquist, B., Lee, S.M., Coker, D.: Medial axis analysis of void structure in threedimensional tomographic images of porous media. J. Geophys. Res. 101B, 8297-8310 (1996). doi:10.1029/95JB03039

Lindquist, W.B., Venkatarangan, A., Dunsmuir, J., Wong, T.F.: Pore and throat size distributions measured from synchrotron X-ray tomographic images of Fontainbleau sandstones. J. Geophys. Res. 105B, 21508 (2000)

Mayer, A.S., Miller, C.T.: The influence of porous medium characteristics and measurement scale on porescale distributions of residual nonaqueous-phase liquids. J. Contam. Hydrol. 11, 189-213 (1992). doi:10.1016/ 0169-7722(92)90017-9

Michalewicz, Z.: Genetic algorithms + data structures = Evolution Programs. AI Series, Springer, New York (1994)

Øren, P.E., Bakke, S.: Process-based reconstruction of sandstones and prediction of transport properties. Transp. Porous Media 46, 311-343 (2002a). doi:10.1023/A:1015031122338

Øren, P.E., Bakke, S.: Reconstruction of Berea Sandstone and pore-scale modeling of wettability effects. In: Proceedings of the 7th International Symposium on Reservoir Wettability, Freycinet, Tasmania (2002b)

Øren, P.E., Bakke, S.: Reconstruction of Berea sandstone and pore-scale modelling of wettability effects. J. Petrol. Sci. Eng. 39, 177-199 (2003). doi:10.1016/S0920-4105(03)00062-7

Øren, P.E., Pinczewski, W.V.: The effect of film flow in the mobilization of waterflood residual oil by gas flooding. In: 6th European IOR-Symposium, Houston, USA (1991)

Øren, P.E., Pinczewski, W.V.: Effect of wettability and spreading on recovery of waterflood residual oil by immiscible gasflooding. SPE Form. Eval. 8, 149-156 (1994)

Øren, P., Billiotte, J., Pinczewski, W.V.: Mobilisation of waterflood residual oil by gas injection of water-wet conditions. SPE Form. Eval. 7, 70-78 (1992). doi:10.2118/20185-PA 
Øren, P.E., Billiotte, J., Pinczewski, W.V.: Pore scale network modelling of waterflood residual oil recovery by immiscible gas flooding. In: SPE Improved Oil Recovery Symposium, Houston, USA (1994)

Øren, P.E., Bakke, S., Arntzen, O.J.: Extending predictive capabilities of network models. SPE J. 3, 324-336 (1998a). doi:10.2118/52052-PA

Øren, P.E., Bakke, S., Arntzen, O.J.: Extending predictive apabilities to network models. SPE J. 3, 324-336 (1998b). doi:10.2118/52052-PA

Paterson, L., Painter, S., Knackstedt, M., Pinczewski, W.V.: Patterns of fluid flow in naturally heterogeneous rocks. Physica A 233, 619-628 (1996a). doi:10.1016/S0378-4371(96)00199-9

Paterson, L., Painter, S., Zhang, X., Pinczewski, W.V.: Simulating residual saturation and relative permeability in heterogeneous formations. In: Proceedings of the 1996 SPE Annual Technical Conference and Exhibition, Denver, Colorado (1996b)

Pereira, G., Pinczewski, W.V., Chan, D., Paterson, L., Øren, P.: Pore-scale network model for drainage dominated three-phase flow in porous media. Transp. Porous Media 24, 167-201 (1996). doi:10.1007/ BF00139844

Reeves, P.C., Celia, M.A.: A functional relationship between capillary pressure, saturation, and interfacial area as revealed by a pore-scale model. Water Resour. Res. 32(8), 2345-2358 (1996). doi:10.1029/ 96WR01105

Renault, P.: The effect of spatially correlated blocking up of some bonds or nodes of a network on the percolation threshold. Transp. Porous Media 6, 451-468 (1991). doi:10.1007/BF00136352

Silin, D.B., Jin, G., Patzek, T.W.: Robust determination of the pore space morphology in sedimentary rocks. In: SPE 84296, Annual Technical Conference and Exhibition, Denver, Colorado, USA (2003)

Sok, R.M., Knackstedt, M.A., Sheppard, A.P., Pinczewski, W.V., Lindquist, W.B., Venkatarangan, A., Paterson, L.: Direct and stochastic generation of network models from tomographic images; effect of topology on two phase flow properties. Transp. Porous Media 46, 345-372 (2002). doi:10.1023/A:1015034924371

Spanne, P., Thovert, J.F., Jacquin, C.J., Lindquist, W.B., Jones, W., Adler, P.M.: Synchrotron computed microtomography of porous media: topology and transports. Phys. Rev. Lett. 73, 2001-2004 (1994). doi:10. 1103/PhysRevLett.73.2001

Thompson, K.E., Willson, C.S., Zhang, W.: Quantitative computer reconstruction of particulate materials from microtomography images. Power Technol. 163, 169-182 (2006). doi:10.1016/j.powtec.2005.12.016

Thovert, J.-F., Salles, J., Adler, P.: Computerised characterization of the geometry of real porous media: their description, analysis and interpretation. J. Microsc. 170, 65-79 (1993)

Wilkinson, D., Willemsen, J.F.: Invasion percolation: a new form of percolation theory. J. Phys. Math. Gen. 16, 3365-3376 (1983). doi:10.1088/0305-4470/16/14/028 